\title{
The deposition coefficient and its role for cirrus clouds
}

\author{
Klaus M. Gierens, ${ }^{1}$ Marie Monier, and Jean-Francois Gayet \\ Laboratoire de Météorologie Physique, Unite Mixte de Recherche, Centre National de la Recherche Scientifique 6016 \\ Université Blaise Pascal, Aubière, France \\ Received 3 December 2001; revised 9 September 2002; accepted 14 November 2002; published 30 January 2003.
}

[1] The potential of homogeneous freezing nucleation to produce large numbers of ice crystals in regions of gentle updraft has been underestimated so far. Small but realistic deposition coefficients for small ice crystals lead to longer periods with relative humidity staying near the critical value for nucleation, which results in much larger numbers of ice crystals than with deposition coefficients close to unity. This mechanism allows to reproduce number densities of crystals in the 10-30 $\mu \mathrm{m}$ size range obtained from recent measurement campaigns. Potential reasons for small deposition coefficients are discussed, and new laboratory experiments to measure them are recommended. INDEX TERMS: 0305 Atmospheric Composition and Structure: Aerosols and particles (0345, 4801); 0320 Atmospheric Composition and Structure: Cloud physics and chemistry; 0399 Atmospheric Composition and Structure: General or miscellaneous; KEYWORDS: ice crystals, crystal growth, homogeneous nucleation, cirrus clouds, deposition coefficient

Citation: Gierens, K. M., M. Monier, and J.-F. Gayet, The deposition coefficient and its role for cirrus clouds, J. Geophys. Res., 108(D2), 4069, doi:10.1029/2001JD001558, 2003.

\section{Introduction}

[2] One of the most uncertain parameters in cloud microphysics is the deposition coefficient, $\alpha$. It controls the growth of ice crystals in the kinetic regime, when crystal size is comparable to the mean free path of air molecules. In the upper troposphere the molecular mean free path is about $0.2 \mu \mathrm{m}$. $\alpha$ is the fraction of water molecules that hit the crystal surface and are incorporated into the crystal lattice. The value of $\alpha$ is very uncertain in particular in the temperature range $-40^{\circ} \mathrm{C}$ to $-70^{\circ} \mathrm{C}$ [see Fukuta and Walter, 1970, Figure 2, or Hobbs, 1974, Figure 6.28], that is, in the upper troposphere where cirrus clouds form. Stephens [1983] mentions experimental values of $\alpha$ ranging from 0.005 to 1 , but under certain conditions it can even be zero for growth along one of the two crystallographic axes [Hallett, 1961]. The initial growth rates of small ice crystals are very sensitive to $\alpha$, and the sensitivity increases with decreasing $\alpha .30 \mathrm{~s}$ of crystal growth can suffice to give differences of one order of magnitude in crystal mass for the range of $\alpha$ given by Stephens [1983].

[3] The deposition coefficient does not only influence crystal growth; it may also affect the formation of cirrus clouds, in particular when homogeneous freezing of solution droplets is the prevalent pathway to ice formation. In such a case the number concentration of ice crystals forming depends strongly on the interplay between supersaturation increase by adiabatic cooling in uplifting air and consumption of water vapor by crystal growth. Heymsfield and Sabin

\footnotetext{
${ }^{1}$ Permanently at Institut für Physik der Atmosphäre, DLR Oberpfaffenhofen, Weßling, Germany.

Copyright 2003 by the American Geophysical Union. 0148-0227/03/2001JD001558\$09.00
}

[1989] already noted that the number of ice crystals produced within their parcel model is sensitive to the choice of kinetic correction factors for vapor diffusion and heat conductivity. Lin et al. [2002] also concluded from a comparison of various cirrus parcel models with $\alpha$ ranging from 0.04 to 1 that the number of ice crystals formed was sensitive to $\alpha$. However, the various models that participated in the comparison are different in many respects. Hence the actual sensitivity remains to be studied.

[4] Although homogeneous ice formation has been considered the main route to cold cirrus since more than 10 years, there appears to persist a tough problem with this theory: The number concentration of ice crystals that form in simulations is smaller than that found in measurements. For instance, Jensen et al. [1994], trying to numerically reproduce First ISCCP (International Satellite Cloud Climatology Project) Regional Experiment (FIRE) intensive field observations, got too few ice crystals in their model, especially at radii less than $50 \mu \mathrm{m}$. They could achieve better agreement only by assuming higher nucleation rates or stronger vertical motions. A survey over some modeling results since 1989 [Heymsfield and Sabin, 1989; Sassen and Dodd, 1989; Jensen and Toon, 1994; DeMott et al., 1994, 1997; Khvorostyanov and Sassen, 1998] shows that for updrafts of $10 \mathrm{~cm} / \mathrm{s}$ or less the number density of ice crystals formed homogeneously is always about some 100 $\mathrm{L}^{-1}$ or less, at least at temperatures that prevail in the midlatitude upper troposphere. Only under tropical tropopause conditions (e.g., $-80^{\circ} \mathrm{C}$ ) higher crystal numbers have been achieved in numerical simulations [Jensen and Toon, 1994].

[5] Evidence of high number densities of small ice particles (i.e. smaller than $20 \mu \mathrm{m}$ ) in cirrus clouds has been presented in several studies [see, e.g., Ström et al., 1997]. More recently, results from the airborne INCA campaign 
(Interhemispheric differences in cirrus properties from anthropogenic emissions [Ström et al., 2000]) definitely suggested that the observation of numerous small ice crystals in cirrus clouds is a common microphysical feature [Gayet et al., 2002]. Typically, about 1 to 5 ice crystals per $\mathrm{cm}^{3}$ are measured even in cirrus formation regions with moderate uplifts of about $10 \mathrm{~cm} / \mathrm{s}$. Such number densities are much larger than those predicted by models.

[6] There are at least three possible reasons for this apparent discrepancy: (1) There could be instrumental effects that feign a much higher ice number concentration than actually exists in reality; (2) Turbulent motions in an overall slow uplift imply higher than average cooling rates locally and lead to higher crystal numbers; and (3) The growth of small, just formed ice crystals could for some reason be slowed down (i.e. the deposition coefficient could be small for small ice crystals). As the discussion has so far concentrated on the first two possibilities, we feel the necessity to discuss how the third one would affect cirrus formation. The three possibilities listed above are by no means mutually exclusive. This means that an investigation of the third one would still be of scientific interest, even if some individual case could be explained by one or both of the other possibilities.

[7] Therefore we pursue in this paper the idea that growth impediments of small ice crystals lead to larger crystal concentrations when a cirrus forms in a slow updraft. We investigate this possibility by varying $\alpha$ in numerical simulations of homogeneous cirrus formation using a parcel model with spectrally resolving microphysics.

\section{Model and Model Equations}

[8] We use the EXMIX model with detailed ice microphysics [Wobrock and Flossmann, 2001] in a parcel model configuration, and we only consider three microphysical processes: the growth of sulfuric acid solution droplets, their homogeneous freezing, and the depositional growth of the resulting ice crystals. Since we consider only the formation of cirrus but not its later evolution, other processes can be ignored.

[9] The model uses 65 logarithmically spaced size classes from $1 \mathrm{~nm}$ to $1.625 \mu \mathrm{m}$ for the $\mathrm{H}_{2} \mathrm{SO}_{4}$ aerosol and 135 classes from $1 \mathrm{~nm}$ to $5 \mathrm{~mm}$ for the ice. The growth of solution droplets is computed explicitly. The nucleation is parameterized after Koop et al. [2000] where we simply set the water activity equal to the saturation ratio with respect to liquid water.

[10] The growth of ice crystals is computed as in the work of Pruppacher and Klett [1997], viz.

$$
\frac{d m}{d t}=\frac{4 \pi C S_{v, i}}{\frac{\rho_{i} R T}{e_{s a t, i} D_{v}^{\prime} M_{w}}+\frac{L_{S} \rho_{i}}{k_{a}^{\prime} T}\left(\frac{L_{S} M_{w}}{R T}-1\right)},
$$

where $C$ is the capacitance factor for the ice crystal; we choose $C$ for a columnar shape. $S_{v, i}$ is the supersaturation with respect to ice, and $e_{s a t, i}$ is the saturation vapor pressure with respect to ice. $\rho_{i}$ is the mass density of the ice crystal. $R, T, M_{w}$, and $L_{S}$ are the universal gas constant, absolute temperature, molecular weight of water, and latent heat of sublimation, respectively. $k_{a}^{\prime}$ is the modified thermal conductivity, and finally, $D_{v}^{\prime}$ is the modified diffusivity of water vapor in air [Pruppacher and Klett, 1997, equation (13-14)]:

$$
D_{v}^{\prime}=\frac{D_{v}}{\frac{r}{r+\Delta_{v}}+\frac{D_{v}}{r \alpha} \sqrt{\frac{2 \pi M_{w}}{R T_{s}}}} .
$$

This correction of the normal diffusivity, $D_{v}$, only accounts for the kinetic effects, but not for ventilation, which is unimportant for small ice crystals and therefore neglected. The terms in the kinetic correction factor are crystal radius $r$, and a "jump" distance $\Delta_{v}$ which can be set equal to the molecular mean free path. $T_{S}$ is the surface temperature of the growing ice crystal and $\alpha$ is the deposition coefficient.

[11] A similar coefficient for the kinetic correction of the heat conductivity, $k_{a}^{\prime}$, that is the thermal accommodation coefficient, has be set to unity [Fukuta and Walter, 1970]. As tests have shown, a change of this factor has negligible influence on the results, at least under upper tropospheric conditions.

\section{Simulation Results}

[12] Simulations with initial conditions: $T_{\text {init }}=-46^{\circ} \mathrm{C}$, $R H_{\text {ice, } \text {,init }}=125 \%$, and pressure $250 \mathrm{hPa}$ are run. The initial distribution of sulfuric acid mass equivalent radius within the aerosol droplets is assumed to be lognormal with number density $N_{a, \text { init }}=150 \mathrm{~cm}^{-3}$, geometric mean radius $10 \mathrm{~nm}$ and geometric standard deviation $\sigma=1.48$. Initially, there is no ice. The model is driven by a $w=10 \mathrm{~cm} / \mathrm{s}$ updraft, which translates into an adiabatic cooling rate of $9.77 \times 10^{-4} \mathrm{~K} / \mathrm{s}$. Each simulation covers a period of $2500 \mathrm{~s}$, which means an uplift of $250 \mathrm{~m}$ and a cooling of $2.4425 \mathrm{~K}$.

[13] Our initial conditions are similar to those of DeMott et al. [1997, Figure 1] although they assumed a power law aerosol size distribution. Consistent with these authors we find freezing nucleation of the solution droplets to occur in a narrow temperature interval at about $-47.7^{\circ} \mathrm{C}$ and at a relative humidity over ice of about $147 \%$. However, whereas DeMott et al. produce about $N_{i}=0.42 \mathrm{~cm}^{-3}$ ice particles, our first simulation assuming $\alpha=1$ gives only $N_{i}=$ $0.039 \mathrm{~cm}^{-3}$ (Figure 1).

[14] Figure 1 shows the strong sensitivity of $N_{i}$ to $\alpha$ and that the sensitivity increases with decreasing $\alpha$. With $\alpha=0.1$ the number of ice crystals is only slightly larger than with $\alpha=1, N_{i}=0.048 \mathrm{~cm}^{-3}$. This weak sensitivity, when $\alpha \geq 0.1$, is consistent with the results of Lin et al. [2002]. However, for $\alpha=0.01$ we get $N_{i}=0.34 \mathrm{~cm}^{-3}$. A further reduction of $\alpha$ leads to quite dramatic increases of $N_{i}$; we get $N_{i}=1.0 \mathrm{~cm}^{-3}$ for $\alpha=0.006, N_{i}=6.2 \mathrm{~cm}^{-3}$ for $\alpha=0.003$, and $N_{i}=83.9$ $\mathrm{cm}^{-3}$ for $\alpha=0.001$, respectively. The error bar in Figure 1 shows the range of $N_{i}$ that was typically found in cirrus clouds under moderate updraft conditions in both INCA campaigns.

[15] Figure 2 shows the ice crystal size spectra at the end of the simulations, when the relative humidity has almost reached ice saturation. The ice mass concentration is determined by ambient humidity, hence it is similar in all experiments. However, the crystal size distributions are rather different. Whereas mode sizes exceed $100 \mu \mathrm{m}$ for $\alpha=1$ and $\alpha=0.1$, they are considerably smaller in the other cases. For $\alpha=0.01$ the mode value yields $90 \mu \mathrm{m}$, and for $\alpha=0.006$ it is $60 \mu \mathrm{m}$. For the two smallest values of $\alpha$ the mode values are $30 \mu \mathrm{m}$ and $12 \mu \mathrm{m}$, respectively. 


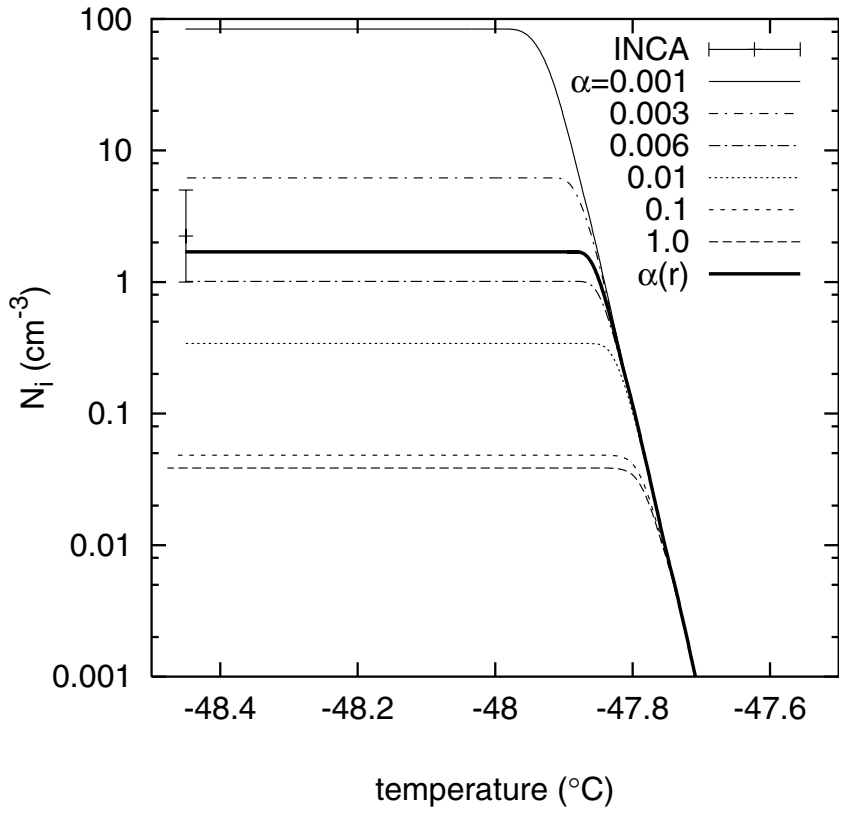

Figure 1. Number of ice crystals frozen homogeneously in a cooling air parcel versus temperature for various values of the deposition coefficient as indicated. For the solid curve a size dependent $\alpha(r)$ was assumed ad hoc: $\alpha(r)=0.359(r)$ $\mathrm{cm})^{0.555}$. A range of typical $N_{i}$ values from INCA is indicated by the error bar.

[16] Although $\alpha=0.001$ is perhaps beyond realistic values for the crystal as a whole (notwithstanding the possibility that it may be zero for certain crystal faces under certain conditions), the dramatic effect on $N_{i}$ and the still considerable effect on the size distributions shows the great potential that growth retardations of small fresh ice crystals can have on the evolution of clouds, and on their microphysical and radiative properties. Possible causes for growth impediments are discussed next.

\section{Discussion}

[17] Aside from being a quantity with a definite physical meaning the deposition coefficient can be considered a tuning parameter from a model viewpoint. Then we must ask whether a turn of this screw is the only possibility to get modeled ice crystal densities in agreement with observed numbers. As field measurements show a wide variability of CCN number concentrations [e.g., Hudson and Xie, 1998] one could try to achieve higher crystal numbers in model results by raising the number of aerosol particles or changing the aerosol size distribution. But this does hardly work. DeMott et al. [1997] surveyed a number of numerical studies [Heymsfield and Sabin, 1989; Jensen and Toon, 1992, 1994a; DeMott et al., 1994; Heymsfield and Miloshevich, 1995] and concluded that effects of the initial aerosol amount and size distribution on $N_{i}$ are insignificant except in very special situations. We have already mentioned that stronger vertical motions, higher nucleation rates, and cold temperatures can also yield larger $N_{i}$ [Jensen and Toon, 1994a; Jensen et al., 1994]. But-now looked at from the physical viewpoint - this all does not allow to derive anything for the deposition coefficient of submicron crystals.
[18] Numerical simulations of ice formation in wave clouds [Jensen et al., 1998] with peak ice number densities of about $4 \mathrm{~cm}^{-3}$ were successful in predicting the observed ice number density without the need for setting the deposition coefficient to small values. At first sight this result seems to contradict the hypothesis we put forward. However, it is necessary to consider the temperature at which this wave cloud formed. This was a rather cold case with $T$ $<-60^{\circ} \mathrm{C}$. A closer inspection of equation (1) shows that the effect of the deposition coefficient gets smaller with decreasing temperature. The growth rate has the form

$$
d m / d t \propto 1 /\left(F_{M}+F_{H}\right),
$$

where $F_{M}$ and $F_{H}$ are temperature dependent functions, and $F_{M}$ depends additionally on the deposition coefficient. A plot of $1 /\left(F_{M}+F_{H}\right)$ versus $T$ shows that the curves for different values of $\alpha$ approach each other at low temperatures. At low $T$ the growth rate of a single crystal becomes small for any value of $\alpha$. Consequently, homogenous freezing of aqueous solution droplets can produce high ice number concentrations at low temperatures, and simulation results of cold cases [Jensen and Toon, 1994; Jensen et al., 1994] agree with this finding.

[19] Unfortunately, laboratory measurements of ice crystal growth rates for submicron sizes under cold $\left(<-40^{\circ} \mathrm{C}\right)$ and substantially supersaturated conditions seem to be unavailable currently. However, there is one measurement at higher temperatures that supports our hypothesis. Anderson and Hallett [1976], observing the depositional growth of ice crystals on substrates of silver iodide (AgI) and covellite $(\mathrm{CuS})$, noticed some delay $(0-400 \mathrm{~s}$, depending on supersaturation and temperature) before the first crystal became visible (size $1 \mu \mathrm{m}$ ) under the microscope after supersaturation had been established. Several possible mechanisms for the

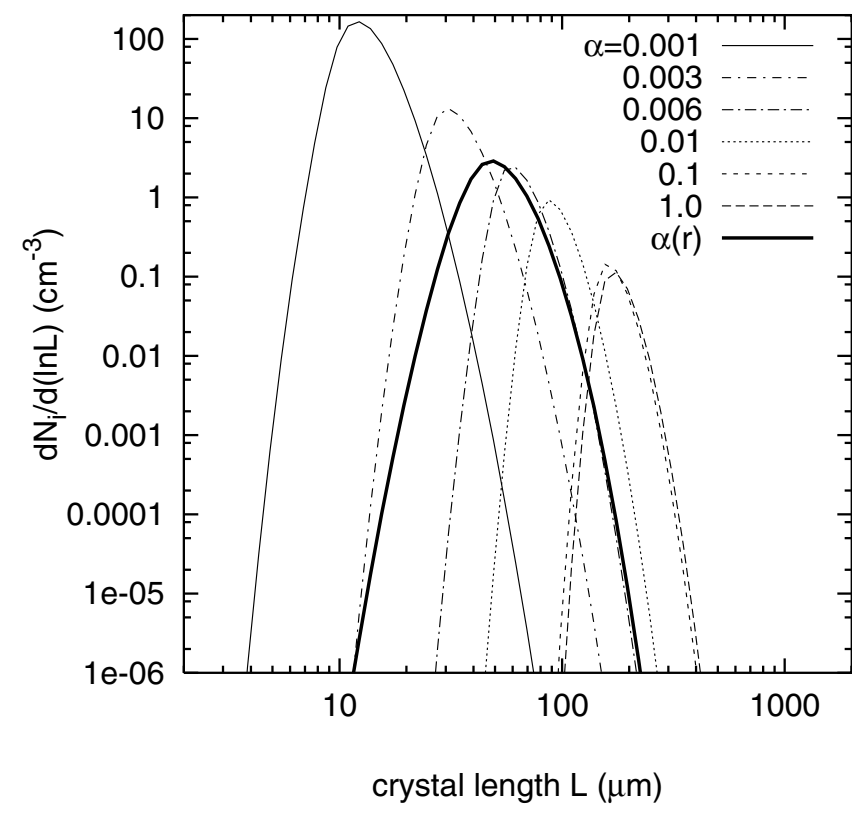

Figure 2. Size spectra of ice crystals frozen homogeneously in a cooling air parcel after $250 \mathrm{~m}$ uplift for various values of the deposition coefficient as indicated. The solid curve is as in Figure 1. 
time lags were discussed, but the only one that worked was the time it takes to grow an ice germ to $1 \mu \mathrm{m}$ size. For $\alpha=0.1$ the growth time was about one second. In order to reproduce longer delays of the order tens of seconds it was necessary to set $\alpha=0.001$. Hence, it cannot be excluded that such small deposition coefficients indeed occur in cirrus clouds.

[20] Theoretical treatments of $\alpha[$ Nelson and Baker, 1996] refer to ice crystals with developed faces that are large compared to the molecular mean free path. It is not clear to us whether and how such theories could be extrapolated to small ice particles that have just been formed. Generally, a water molecule approaching the crystal surface from the vapor phase must find a crystallographic dislocation in order to be incorporated permanently into the crystal lattice. At such a defect (a ledge) it is much easier for a water molecule to find an open place in the crystal lattice than on a smooth surface (a terrace). Only a fraction $\alpha$ of the approaching water molecules find their way to a ledge and make it to the crystal. There are two possibilities: (1) the surface density of ledges is similar for small and large ice crystals, which would imply a small number of ledges on small crystals; (2) the number of ledges is similar on small and large crystals, which would imply a higher surface density of ledges on small crystals. In the latter case there is the more competition between the ledges for the approaching water molecules the smaller the crystal is. $\alpha$ should decrease with decreasing crystal size in both cases. Simulations with size dependent $\alpha$ can give high $N_{i}$ and small mode sizes as exemplified in Figures 1 and 2.

[21] Surface impurities may also hinder the migration of water molecules to the ledges. Sassen et al. [1995] mention that, while a liquid sulfuric acid solution droplet freezes homogeneously, the acid can be excluded to the surface, producing a liquid coating of sulfuric acid on the fresh crystal. It is conceivable that such or similar coatings slow down the molecule's motion on the surface and delay the growth of small ice crystals.

[22] One also might argue freezing of a highly supercooled droplet would yield a highly polycrystalline pristine ice particle with such a small scale roughness, that it would imply a large deposition coefficient at the beginning of depositional growth. However, this is improbable. One can estimate the time it needs for a $0.1 \mu \mathrm{m}$ droplet to completely freeze after the first supercritical ice germ has been formed. We take for an estimation the usual growth equation in a simple form (neglecting both the solute and the release of latent heat):

$$
r_{i}\left(d r_{i} / d t\right)=D \Delta \rho / \rho_{i}
$$

where $r_{i}$ is the radius of the small ice germ that has just been formed, $D \approx 10^{-5} \mathrm{~cm}^{2} / \mathrm{s}$ is the diffusion coefficient of water molecules in liquid water, $\rho_{i}$ is the bulk density of ice $\left(\rho_{i} \approx\right.$ $0.9 \mathrm{~g} \mathrm{~cm}^{-3}$ ), and where we take the density difference of water molecules in the liquid droplet and the ice crystal for $\Delta \rho$, i.e. $\Delta \rho \approx 0.1 \mathrm{~g} \mathrm{~cm}^{-3}$. Integration of this equation (from zero to the drop radius $r_{d}$ ) gives the time $\tau$ for complete freezing as:

$$
\tau=\frac{r_{d}^{2} \rho_{i}}{2 D \Delta \rho} \approx 5 \times 10^{-5} \mathrm{~s}
$$

for a $r_{d}=0.1 \mu \mathrm{m}$ droplet. Thus, when the first supercritical ice embryo has been formed, another one must form within $10^{-5} \mathrm{~s}$ or so in order to produce a polycrystal out of the droplet. Polycrystals therefore could only make a substantial fraction of the homogeneously frozen droplets if the nucleation rate would reach a value of 1 event per $(0.1$ $\mu \mathrm{m})^{3}$ per $10^{-5}$ s, i.e. $J \approx 10^{20} \mathrm{~cm}^{-3} \mathrm{~s}^{-1}$. We have made some simple box model calculations (simulating homogeneous freezing nucleation in an uplifting air mass) and have calculated the maximum $J$. In all cases (up to an uplift of $10 \mathrm{~m} / \mathrm{s}$ ) it was many orders of magnitude less than $10^{20}$ $\mathrm{cm}^{-3} \mathrm{~s}^{-1}$. Thus, we conclude that the formation of polycrystalline structure is improbable during the homogeneous freezing of aqueous solution droplets of submicron size. However, since the necessary nucleation rate for the nearly simultaneous formation of more than one ice germ varies with $r_{d}^{-5}$, such a process may occur in drops larger than a few micron. This could explain the frequent formation of polycrystals from freezing of such drops (see examples in chapter 2 of Pruppacher and Klett [1997]).

[23] The vastly different measured values of $\alpha$, even for similar temperatures, could result from measurements with crystals of different size. If the number of effective ledges decreases with decreasing crystal size, measurements of $\alpha$ will depend on crystal size. The possibility of size effects seems not to be noticed so far in the literature. To our opinion it is worth to look at such effects.

\section{Conclusions}

[24] A principle study on the effect of the deposition coefficient, $\alpha$, onto the number of ice crystals, $N_{i}$, forming homogeneously in a weak updraft shows that $N_{i}$ increases strongly with decreasing $\alpha$ and that the sensitivity is the stronger the smaller is $\alpha$. Current microphysical parcel models seem to prefer $0.1 \leq \alpha \leq 1.0$, but $N_{i}$ varies little in this range in our results. Experimental values of $\alpha$ extend to values as low as 0.005 in a temperature range from -40 to $-70^{\circ} \mathrm{C}$. In our calculation a reduction of $\alpha$ to 0.01 and less leads to dramatic increases of $N_{i}$, because the just formed crystals then do not effectively reduce the supersaturation by deposition growth, thus the nucleation process can proceed for a longer period at high rates.

[25] Although experimental verification of $\alpha<0.01$ for submicron ice crystals seems to be missing, this hypothesis is able to explain recent measurements of large number densities of small ice crystals $(\$ 20 \mu \mathrm{m})$ in regions with weak updrafts $(w \approx 10 \mathrm{~cm} / \mathrm{s})$.

[26] The deposition coefficient may depend on crystal size. $\alpha$ could be small for submicron crystals because either the number of ledges on the ice surface where water molecules can attach decreases with decreasing crystal size, or because the ledges approach each other on a small surface and compete for the approaching gas molecules. Both these possibilities, and the additional possibility of surface impurities could result in lower growth rates for submicron ice crystals.

[27] Since experimental evidence of this hypothetical, but potentially important, mechanism is missing, efforts should be undertaken to settle this point. The deposition coefficient is still an unknown quantity after decades of cloud microphysics research, and the new findings from the recent 
campaigns are a good occasion to turn attention back to this perhaps underestimated factor.

[28] Acknowledgments. The authors thank A. Flossmann and W. Wobrock for critically reading the manuscript. Two reviewers raised interesting points to discuss that were missing in the original manuscript. KMG thanks CNRS for the possibility to work at LaMP. This work contributes to the project GI 333/1-1 of the Deutsche Forschungsgemeinschaft. JFG thanks the EU for financial support within INCA (EVK2-CT1999-00039).

\section{References}

Anderson, B. J., and J. Hallett, Supersaturation and time dependence of ice nucleation from the vapor on single crystal substrates, J. Atmos. Sci., 33, $822-832,1976$.

DeMott, P. J., M. P. Meyers, and W. R. Cotton, Numerical model simulations of cirrus clouds including homogeneous and heterogeneous ice nucleation, J. Atmos. Sci., 51, 77-90, 1994.

DeMott, P. J., D. C. Rogers, and S. M. Kreidenweis, The susceptibility of ice formation in upper tropospheric clouds to unsoluble aerosol components, J. Geophys. Res., 102, 19,575-19,584, 1997.

Fukuta, N., and L. A. Walter, Kinetics of hydrometeor growth from a vaporspherical model, J. Atmos. Sci., 27, 1160-1172, 1970.

Gayet, J.-F., F. Auriol, A. Minikin, J. Ström, M. Seifert, R. Krejci, A. Petzold, G. Febvre, and U. Schumann, Quantitative measurement of the microphysical and optical properties of cirrus clouds with four different in situ probes: Evidence of small ice crystals, Geophys. Res. Lett., 29(24), 2230, doi:10.1029/2001GL0014342, 2002.

Hallett, J., The growth of ice crystals on freshly cleaved covellite surfaces, Philos. Mag., 6, 1073-1087, 1961 .

Heymsfield, A. J., and L. M. Miloshevich, Relative humidity and temperature influences on cirrus formation and evolution: Observations from wave clouds and FIRE II, J. Atmos. Sci., 52, 4302-4326, 1995.

Heymsfield, A. J., and R. M. Sabin, Cirrus crystal nucleation by homogeneous freezing of solution droplets, J. Atmos. Sci., 46, 2252-2264, 1989.

Hobbs, P. V., Ice Physics, 837 pp., Oxford Univ. Press, New York, 1974.

Hudson, J. G., and Y. Xie, Cloud condensation nuclei measurements in the high troposphere and in jet aircraft exhaust, Geophys. Res. Lett., 25, 1395-1398, 1998.

Jensen, E. J., and O. B. Toon, The potential effects of volcanic aerosols on cirrus, Geophys. Res. Lett., 19, 1759-1762, 1992.

Jensen, E. J., and O. B. Toon, Ice nucleation in the upper troposphere: Sensitivity to aerosol composition and size distribution, temperature, and cooling rate, Geophys. Res. Lett., 21, 2019-2022, 1994.

Jensen, E. J., O. B. Toon, D. L. Westphal, S. Kinne, and A. J. Heymsfield, Microphysical modeling of cirrus, 1, Comparison with 1986 FIRE IFO measurements, J. Geophys. Res., 99, 10,421-10,442, 1994.
Jensen, E. J., et al., Ice nucleation processes in upper tropospheric waveclouds observed during SUCCESS, Geophys. Res. Lett., 25, 1363-1366, 1998.

Khvorostyanov, V. I., and K. Sassen, Cirrus cloud simulation using explicit microphysics and radiation, part II, Microphysics, vapor and ice mass budgets, and optical and radiative properties, J. Atmos. Sci., 55, $1822-$ $1845,1998$.

Koop, T., B. Luo, A. Tsias, and T. Peter, Water activity as the determinant for homogeneous ice nucleation in aqueous solutions, Nature, 406, $611-$ 614, 2000.

Lin, R. F., D. O’C. Starr, P. J. DeMott, R. Cotton, K. Sassen, E. Jensen, B. Kärcher, and X. Liu, Cirrus parcel model comparison project, part I, The critical components to simulate cirrus initiation explicitly, J. Atmos. Sci., 59, 2305-2329, 2002.

Nelson, J. T., and M. B. Baker, New theoretical framework for studies of vapor growth and sublimation of small ice crystals in the atmosphere, J. Geophys. Res., 101, 7033-7047, 1996.

Pruppacher, H. R., and J. D. Klett, Microphysics of Clouds and Precipitation, 2nd ed., 954 pp., Kluwer Acad., Norwell, Mass., 1997.

Sassen, K., and G. C. Dodd, Homogeneous nucleation rate for highly supercooled cirrus cloud droplets, J. Atmos. Sci., 45, 1357-1369, 1989.

Sassen, K., D. O’C. Starr, G. G. Mace, M. R. Poellot, S. H. Melfi, W. L. Eberhard, J. D. Spinhirne, E. W. Eloranta, D. E. Hagen, and J. Hallett, The 5-6 December 1991 FIRE IFO II jet stream cirrus case study: Possible influences of volcanic aerosols, J. Atmos. Sci., 52, 97-123, 1995.

Stephens, G. L., The influence of radiative transfer on the mass and heat budgets of ice crystals falling in the atmosphere, J. Atmos. Sci., 40, 1729-1739, 1983.

Ström, J., B. Strauss, F. Schröder, T. Anderson, J. Heintzenberg, and P. Wendling, In situ observations of microphysical properties of young cirrus, J. Atmos. Sci, 54, 2542-2553, 1997.

Ström, J., F. Flatøy, J.-F. Gayet, M. Kulmala, J. Ovarlez, and U. Schumann, Observations in cirrus clouds during the INCA Southern Hemisphere campaign, in Proceedings of the International Conference on Clouds and Precipitation, Reno NV, 14-18 August 2000, pp. 1207-1208, 2000.

Wobrock, W., and A. I. Flossmann, 2000: Modelling of the formation of cirrus clouds, in Aviation, Aerosols, Contrails and Cirrus Clouds (A2C3), Proceedings of a European Workshop, Seeheim (Near Frankfurt/Main), Germany, July 10-12, 2000, Air Pollut. Res. Rep. 74, EUR 19428, edited by U. Schumann and G. T. Amanatidis, p. 314, Eur. Comm., Brussels, 2001 .

J.-F. Gayet, K. M. Gierens, and M. Monier, Laboratoire de Météorologie Physique, UMR Centre National de la Recherche Scientifique 6016 , Université Blaise Pascal, 24, av. des Landais, F-63177, Aubière, France. (klaus.gierens@dlr.de) 